\title{
A first survey of the rye (Secale cereale) genome composition through BAC end sequencing of the short arm of chromosome IR
} Jan Bartoš*1, Etienne Paux², Robert Kofler ${ }^{3}$, Miroslava Havránková1, David Kopecký1 ${ }^{1}$ Pavla Suchánková1, Jan Šafářr ${ }^{1}$, Hana Šimková1, Christopher D Town ${ }^{4}$, Tamas Lelley ${ }^{3}$, Catherine Feuillet ${ }^{2}$ and Jaroslav Doležel ${ }^{1,5}$

\author{
Address: ${ }^{1}$ Laboratory of Molecular Cytogenetics and Cytometry, Institute of Experimental Botany, Sokolovská 6, CZ-77200 Olomouc, Czech \\ Republic, ${ }^{2}$ INRA- Université Blaise Pascal, UMR GDEC 1095, 234 Avenue du Brezet, F-63100 Clermont-Ferrand, France, ${ }^{3}$ University of Natural \\ Resources and Applied Life Sciences, Department for Agrobiotechnology, Institute for Plant Production Biotechnology, Konrad Lorenz Str. 20, A- \\ 3430 Tulln, Austria, ${ }^{4}$ The J. Craig Venter Institute, 9704 Medical Center Drive, Rockville MD 20850, USA and ${ }^{5}$ Department of Cell Biology and \\ Genetics, Palacký University, Šlechtitelù 11, CZ-78371 Olomouc, Czech Republic \\ Email: Jan Bartoš* - bartos@ueb.cas.cz; Etienne Paux - etienne.paux@clermont.inra.fr; Robert Kofler - robert.kofler@boku.ac.at; \\ Miroslava Havránková - MiroslavaHavrankova@seznam.cz; David Kopecký - kopecky@ueb.cas.cz; Pavla Suchánková - suchankova@ueb.cas.cz; \\ Jan Šafár - safar@ueb.cas.cz; Hana Šimková - simkovah@ueb.cas.cz; Christopher D Town - cdtown@jcvi.org; \\ Tamas Lelley - tamas.lelley@boku.ac.at; Catherine Feuillet - feuillet@clermont.inra.fr; Jaroslav Doležel - dolezel@ueb.cas.cz \\ * Corresponding author
}

Published: 19 September 2008

BMC Plant Biology 2008, 8:95 doi:10.1 I86/147|-2229-8-95
Received: 15 May 2008

Accepted: 19 September 2008

This article is available from: http://www.biomedcentral.com/I47I-2229/8/95

(C) 2008 Bartoš et al; licensee BioMed Central Ltd.

This is an Open Access article distributed under the terms of the Creative Commons Attribution License (http://creativecommons.org/licenses/by/2.0), which permits unrestricted use, distribution, and reproduction in any medium, provided the original work is properly cited.

\begin{abstract}
Background: Rye (Secale cereale L.) belongs to tribe Triticeae and is an important temperate cereal. It is one of the parents of man-made species Triticale and has been used as a source of agronomically important genes for wheat improvement. The short arm of rye chromosome I (IRS), in particular is rich in useful genes, and as it may increase yield, protein content and resistance to biotic and abiotic stress, it has been introgressed into wheat as the IBL.IRS translocation. A better knowledge of the rye genome could facilitate rye improvement and increase the efficiency of utilizing rye genes in wheat breeding.

Results: Here, we report on BAC end sequencing of I,536 clones from two IRS-specific BAC libraries. We obtained 2,778 (90.4\%) useful sequences with a cumulative length of 2,032,538 bp and an average read length of 732 bp. These sequences represent $0.5 \%$ of IRS arm. The GC content of the sequenced fraction of IRS is $45.9 \%$, and at least $84 \%$ of the IRS arm consists of repetitive DNA. We identified transposable element junctions in BESs and developed insertion site based polymorphism markers (ISBP). Out of the 64 primer pairs tested, 17 (26.6\%) were specific for IRS. We also identified BESs carrying microsatellites suitable for development of IRS-specific SSR markers.
\end{abstract}

Conclusion: This work demonstrates the utility of chromosome arm-specific BAC libraries for targeted analysis of large Triticeae genomes and provides new sequence data from the rye genome and molecular markers for the short arm of rye chromosome I. 


\section{Background}

Rye (Secale cereale L.) is a temperate cereal belonging to the tribe Triticeae, which is grown mainly in Europe and Northern America. Its uses include grain, hay, pasture, cover crop, green fodder, and green manure. More than $50 \%$ of the annual rye harvest is used for bread making, resulting in rich, dark bread that holds its freshness for about a week. Despite its relatively low acreage compared to other cereals, rye is of great importance due to its broad tolerance to biotic and abiotic stress, a feature generally lacking in other temperate cereals. Thus, rye remains an important grain crop species for cool temperate zones.

Besides its importance as a crop, rye is one of the parents of a man-made species Triticale and the short arm of rye chromosome 1 (1RS) has been introgressed into several hundreds of wheat cultivars [1,2]. In fact, some of the most successful wheat varieties carry the 1BL.1RS translocation as the presence of $1 \mathrm{RS}$ in the wheat genome increases both yield and protein content in grains [3]. Moreover, 1RS carries a cluster of genes encoding resistance to stem, leaf and yellow rust - Sr31, Lr26 and Yr9, respectively [4] and a self-incompatibility locus [5]. On the down side, 1RS carries the Sec-1 locus coding for $\varepsilon$-secalin, which negatively affects bread making quality [6]. Thus, it would be of great advantage to isolate those genes individually through map-based cloning and develop markers for marker assisted selection in rye and wheat.

Despite the economic importance of rye, little is known about its genetic make up at the DNA sequence level. To our knowledge, there is no ongoing sequencing project in rye, and there are no plans to target gene-rich fractions of its genome. Rye is underrepresented in the sequence databases compared to wheat and barley for which 1,104,002 and 529,839 sequences respectively, are deposited in GenBank. There are only 9,807 rye sequences (about $5 \mathrm{Mbp}$ ) available, of which about $90 \%$ are expressed sequence tags (ESTs). Updated list of rye genes, markers and linkage data was created by Schlegel and Korzun [7]. The lack of sequence information is a major limitation for marker development and gene cloning in this species.

The monoploid genome size of rye $(1 \mathrm{Cx}=7,917 \mathrm{Mbp})$ is the largest among temperate cereals, almost 40\% larger than that of bread wheat (Table 1). This is due to the presence of a large amount of highly repetitive sequences. Flavell et al. [8] estimated the repetitive DNA content of rye to be $92 \%$. Despite the progress in sequencing technology and bioinformatics, sequencing the whole rye genome remains a very difficult and expensive task. In particular, genome shotgun sequencing of such a large and repetitive genome seems currently impossible. On the other hand, the short arm of rye chromosome 1 represents only 5.6\% of the rye genome and with the molecular size of 441 $\mathrm{Mbp}, 1 \mathrm{RS}$ is comparable in size to the whole rice genome, which was recently sequenced [9-11]. Recently, a method has been developed to dissect large plant genomes into individual chromosomes using flow cytometric sorting (reviewed in $[12,13]$ ). A protocol for sorting individual rye chromosomes has been set up by Kubaláková et al. [14], and Šimková et al. [15] created two BAC libraries from flow sorted 1RS arms. The library represents a valuable tool for map-based cloning, targeted sequencing and marker development.

End sequencing of BAC clones enables generating random sequence information distributed across the whole genome. Kelley et al. [16] developed a protocol for high throughput BAC end sequence (BES) generation using automated sequencers. This protocol is now a routine in large sequencing centers, reducing cost and enabling the creation of large data sets. Nevertheless, the number of BESs from the Triticeae tribe is currently limited with only 37,609 hexaploid wheat BESs and 32 Triticum monococcum BESs in GenBank, representing the whole tribe. Beyond the sequence information itself, BESs are a valuable source of molecular markers. Shultz et al. [17] used BESs derived from BACs, representing minimum tiling path of soybean, to develop new microsatellite markers. Among the first 135 primer pairs tested, more than 60\% were polymorphic. Paux et al. [18] took advantage of a BAC library specific for wheat chromosome 3B [19], and sequenced BAC ends to isolate chromosome-specific molecular markers based on inserted transposable elements (ISBP - Insertion Site Based Polymorphism). Paux et al. [18] succeeded in developing thirty-nine 3B-specific markers to anchor BAC contigs to the genetic/deletion map and have since then developed several hundreds of ISBP markers from 3B

Table I: Genome size of major Triticeae species

\begin{tabular}{llccc}
\hline Species & Chromosome number & IC genome size & ICx genome size* & Reference \\
\hline Rye & $2 n=2 x=14(\mathrm{RR})$ & $7,917 \mathrm{Mbp}$ & $7,917 \mathrm{Mbp}$ & {$[51]$} \\
Durum wheat & $2 n=4 x=28(\mathrm{AABB})$ & $12,030 \mathrm{Mbp}$ & $6,015 \mathrm{Mbp}$ & {$[52]$} \\
Bread wheat & $2 n=6 x=42(\mathrm{AABBDD})$ & $16,937 \mathrm{Mbp}$ & $5,646 \mathrm{Mbp}$ & {$[52]$} \\
Barley & $2 n=2 x=14(\mathrm{HH})$ & $5,100 \mathrm{Mbp}$ & $5,100 \mathrm{Mbp}$ & {$[51]$} \\
\hline
\end{tabular}

*Monoploid genome size (see [53] for terminology on genome size) 
(unpublished). According to the authors' estimate, about $5 \%$ of BESs are suitable for ISBP development.

Here, we report on DNA sequence composition of the short arm of rye chromosome 1 (1RS) and on the development of new molecular markers for this chromosome arm using $2 \mathrm{Mb}$ of BAC end sequences. We demonstrate that the combination of chromosome arm-specific $\mathrm{BAC}$ library with BAC end sequencing technology offers a cost efficient strategy to survey the composition of the rye genome and saturate chromosome 1RS with molecular markers.

\section{Methods}

\section{Plant material}

Seeds of rye (Secale cereale L., $2 \mathrm{n}=14) \mathrm{cv}$. Imperial and wheat (Triticum aestivum L., $2 \mathrm{n}=42$ ) $\mathrm{cv}$. Chinese Spring were kindly provided by Prof. A. J. Lukaszewski (University of California, Riverside, USA). Seeds of barley (Hordeum vulgare $\mathrm{L} ., 2 \mathrm{n}=14) \mathrm{cv}$. Akcent were obtained from Dr. P. Martínek (Agriculture Research Institute, Kroměříž, Czech Republic). A ditelocentric 1RS wheat-rye (Chinese Spring - Imperial) addition line $(2 \mathrm{n}=42+1 \mathrm{RS}$ ") was obtained from Dr. B. Friebe (Kansas State University, Manhattan, USA).

\section{IRS-specific BAC libraries}

Two BAC libraries specific for the short arm of rye chromosome 1 were constructed from DNA of 1RS arms, which were flow-sorted from the above mentioned wheatrye ditelocentric 1RS addition line [15]. The SccImp1RShA library (HindIII) consists of 66,816 clones with an average insert size of $72 \mathrm{~kb}$ ordered in $174 \times 384$ well plates. The SccImp1RSbA library (BamHI) consists of 36,864 clones with an average insert size of $75 \mathrm{~kb}$ ordered in $96 \times 384$-well plates. Collectively both libraries cover the chromosome arm 14-fold. As the 1RS arms were sorted from wheat alien chromosome addition line, the libraries contain about $14 \%$ of clones from various wheat chromosomes [15].

\section{BAC end sequencing \\ Two plates from each BAC library (plates SccImp1RShA_0079, SccImp1RShA_0127, SccImp1RSbA_0175 and SccImp1RSbA_0223) were cho- sen randomly for BAC end sequencing. DNA templates were prepared in 384-well format by a standard alkaline lysis method. The end sequencing was performed using Applied Biosystems (ABI) Big Dye terminator chemistry and analyzed on ABI 3730xl sequencer. Base calling was performed using TraceTuner and sequences were trimmed for vector and low quality sequences using Lucy [20].}

\section{Annotation of sequences}

Three repeat databases were used to analyze the repetitive fraction of the BAC-end sequences: TREPtotal [21,22], RepBase [23,24] and TIGR Plant Repeat Databases $[25,26]$. For identification of genes in BESs, 960,574 PUTs (PlantGDB-assembled Unique Transcripts) from various plant species were used. PUTs of Arabidopsis thaliana $(143,848)$, Avena sativa $(5,595)$, Brachypodium distachyon (9,924), Glycine max $(102,265)$, Hordeum vulgare $(103,345)$, Oryza sativa $(153,740)$, Secale cereale $(5,976)$, Sorghum bicolor $(44,953)$, Triticum aestivum $(243,326)$, Triticum monococcum $(6,986)$ and Zea mays $(140,616)$ were downloaded from PlantGDB $[27,28]$.

\section{Identification of repetitive DNA elements}

A semi-automated pipeline [18] was used to search for repetitive DNA elements in BAC-end sequences. The procedure involved two steps to find known repeats in a sequence and an additional step identifying potential repeats. In the first step, RepeatMasker [29] with the CrossMatch algorithm and default settings was used to search repeats without specifying a custom library. Thereafter, sequences were searched against TREPtotal, RepBase and TIGR Plant Repeat Databases. In the second step sequences were searched using TBLASTX (E-value $=1 \mathrm{e}^{-5}$ ) [30] against the same databases. Sequences matching known repeats were masked with an "X". Putative unknown repeats were identified by searching masked BESs with BLASTN [30] against themselves and 32,496 genome survey sequences (GSSs) of Triticum and Aegilops spp. downloaded from GenBank [31]. Sequences displaying $80 \%$ identity over at least 100 bp and five matches were assumed as unknown repeats and masked with an "X". The fraction of genome, represented by each repetitive DNA element, was calculated as ratio of cumulative length of sequences with homology to the element and the total length of BES data set.

\section{Gene content analysis}

The repeat masked sequences were subjected to a homology search using BLASTN (E-value $=1 \mathrm{e}^{-30}$ ) against the PUT collections mentioned above. Cumulative match length was used to calculate the fraction of coding sequences in the rye genome as described for repetitive elements. Sequences matching PUTs coding for TE-related proteins were omitted. Sequences with alignment longer than 200 bp were searched using BLASTX against non-redundant protein sequences (with default setting except E-value = $\left.1 \mathrm{e}^{-10}\right)$.

\section{Development of molecular markers}

We used SciRoKo 3.3 computer program [32] with default settings (except minimum score 14) for the identification of microsatellites in the BAC end sequences. BESs containing junction between two different sequences (repetitive 
elements or repetitive element and non-repetitive sequence) were identified from repeat-masking analysis for development of ISBP markers. Primer pairs were designed using PRIMER3 software [33] with default settings to border corresponding junction.

\section{Chromosome sorting and DNA amplification}

Ten thousand $1 \mathrm{R}$ chromosomes were sorted from rye $\mathrm{cv}$. Imperial according to Kubaláková et al. [14] into $20 \mu \mathrm{l}$ $\mathrm{ddH}_{2} \mathrm{O}$ using a FACSVantage SE cell sorter (Becton Dickinson). Twenty thousand all rye chromosomes except $1 \mathrm{R}$ $(2 \mathrm{R}-7 \mathrm{R})$ were sorted in the same way (Figure 1). Chromosomal DNA obtained after proteinase treatment was amplified using GenomiPhi DNA Amplification Kit (GE Healthcare, UK) according to the manufacturer's instruction to obtain $5 \mu \mathrm{g}$ chromosome-specific DNA.

\section{Physical mapping of molecular markers}

Sixty four ISBP primer sets were tested for 1RS specificity. For this purpose PCR was carried out on several DNA templates: a - rye (cv. Imperial); b - wheat (cv. Chinese Spring); c -wheat-rye (Chinese Spring - Imperial) telocentric 1RS addition; $\mathrm{d}$ - flow-sorted chromosome 1R; e flow-sorted chromosomes 2R - 7R. Genomic DNA was isolated using Invisorb Spin Plant Mini Kit (Invitek) according to the manufacturer's instruction. The $10 \mu \mathrm{l}$ standard PCR reaction contained 25 ng DNA, $1 \times$ PCR buffer, $0.01 \%$ Cresol Red, $1.5 \%$ sucrose, $0.2 \mathrm{mM}$ each of dNTPs, $5 \mu \mathrm{M}$ primers, $0.5 \mathrm{U}$ Taq DNA polymerase. PCR was performed in a PTC-200 thermal cycler (MJ Research)

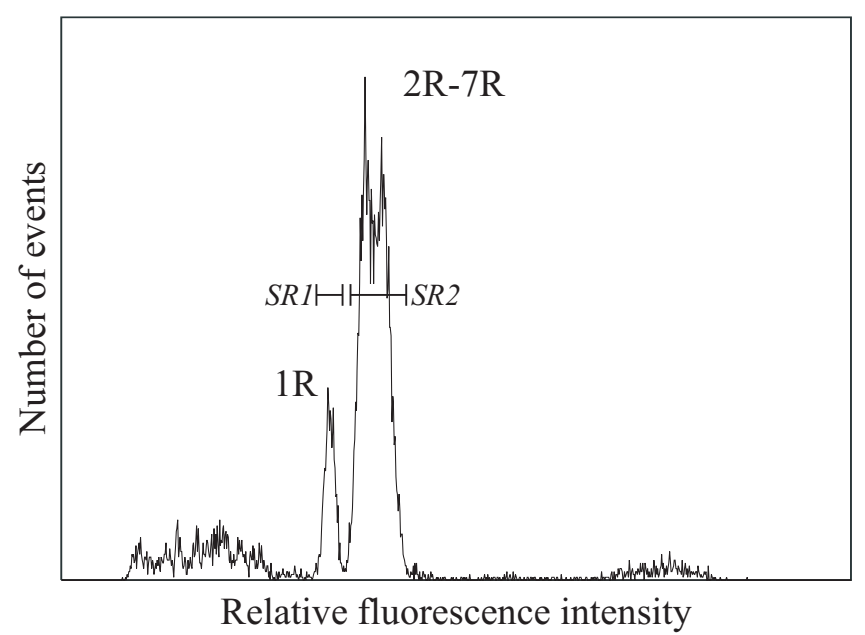

Figure I

Flow cytometric chromosome analysis and sorting in rye. Histogram of relative fluorescence intensity obtained after analysis of suspension of DAPI-stained rye chromosomes. Peak of chromosome IR is clearly resolved from the composite peak of chromosomes 2R-7R. Sorting regions were set to separate chromosome IR (SRI) from other chromosomes (SR2). as follows: initial denaturation at $95^{\circ} \mathrm{C}$ for $5 \mathrm{~min}$; 35 cycles of $95^{\circ} \mathrm{C}$ for $30 \mathrm{sec}, 62^{\circ} \mathrm{C}$ for $30 \mathrm{sec}, 72^{\circ} \mathrm{C}$ for $30 \mathrm{sec}$; final extension for 5 minutes at $72^{\circ} \mathrm{C}$. PCR products were separated on $2 \%$ agarose gel.

\section{Localization of a new repeat by Fluorescence in situ Hybridization (FISH)}

To localize a newly identified repeat on mitotic metaphase chromosomes of rye, wheat and barley, root tips were collected to ice water for 26-30 h, fixed in a mixture of absolute alcohol: glacial acetic acid $(3: 1)$ at $37^{\circ} \mathrm{C}$ for seven days and stored at $-18^{\circ} \mathrm{C}$. Cytological preparations and in situ hybridization with labeled DNA were made according to Massoudi-Nejad et al. [34]. Digoxigeninlabeled probe was prepared from the newly identified COP1 repeat by PCR with specific primers (Left - CAACATTCGTGATGGTTTCG; Right - ATACACAAACCTGCCCCAAA). For identification of wheat homoeologous groups, reprobing with two additional probes was done. Biotin-labeled probe for GAA microsatellites was prepared using PCR with $(\mathrm{GAA})_{7}$ and $(\mathrm{CCT})_{7}$ primers and wheat genomic DNA as a template. A probe for 260-bp fragment of the Afa family repeat was prepared and labeled by digoxigenin using PCR with primers AS-A and AS-B on wheat genomic DNA according to Kubaláková et al. [35]. Chromosomes were counterstained with $1.5 \mu \mathrm{g} /$ $\mathrm{ml}$ 4',6-diamidino-2-phenylindole (DAPI). Hybridization signal was visualized with anti-digoxigenin-fluorescein and Cy3-labeled streptavidin and observed under a fluorescence microscope (Olympus AX70 with a SensiCam B/ W CCD camera attached).

\section{Results \\ $B A C$ end sequencing and sequence trimming}

Four 384-well plates originating from two BAC libraries specific for rye chromosome arm 1RS were chosen to provide a uniform and random sample of $1 \mathrm{RS}$. BAC clones were sequenced from both ends and after trimming, 2,778 (90.4\%) useful sequences (longer than $60 \mathrm{bp}$ ) were obtained. In total, 2,032,538 bp of 1RS specific sequences were generated with average read length of $732 \mathrm{bp}$. These sequences represent $0.5 \%$ DNA of the short arm of rye chromosome 1. Analysis of the GC content of the sequenced fraction of $1 \mathrm{RS}$ is showed a $45.9 \%$ composition. All sequences were deposited in GenBank (Accession numbers FI104352-핌129).

\section{Identification and characterization of repetitive DNA elements}

The cumulative length of the sequences with homology to a certain repetitive element was used to estimate its representation in the rye genome. For example, sequences with homology to Copia retroelements had a cumulative length of $281,937 \mathrm{bp}$ representing $13.9 \%$ of the BESs $(2,032,538 \mathrm{bp})$. Should BESs composition be representa- 
tive for rye, Copia elements account for $13.9 \%$ of the whole rye genome (Table 2).

In total, $75.6 \%$ of the data set showed homology to repeats deposited in the databases listed above. Retrotransposons (Class I elements) were the dominant repeat group in the analyzed sequences, comprising $64.3 \%$ of sequencing data. In contrast, class II elements (DNA transposons) constituted only a minor part of 1 RS $(5.0 \%)$. Almost the same fraction of the sequences analyzed $(4.7 \%)$ showed high similarity to ribosomal RNA genes. This is consistent with the presence of the nucleolar organizing region (NOR) on 1RS.

Searching BES with masked repeats against themselves and genome survey sequences (GSSs) from Triticum and Aegilops spp., identified 178,027 bp ( $8.8 \%$ of the data set) as unknown repetitive elements. However, the BESs were generally not long enough (average length $732 \mathrm{bp}$ ) to cover complete units of the newly identified repeats and allow the identification of new elements. Thus, most of the sequences could not be further characterized. Only one repeat, COP1 with a unit length of about 500 bp could be further characterized. Two BESs, SccImp1RShA_0079_A17F [GenBank:FI104367] and SccImp1RShA_0079_J11F [GenBank:FI104773], contained each one complete and one partial unit of this repeat with identity ranging from 85 to $95 \%$. These sequences were used for multiple alignment (ClustalW with default settings) and consensus sequence calculation (Figure 2). Finally, a BLAST search (E-value $=1 \mathrm{e}^{-10}$ ) against the complete BES data set revealed eight complete or partial units. Assuming that our data set represents $0.5 \%$ of $1 \mathrm{RS}$, one can estimate that there is more than 1,000 COP1 units in chromosome 1RS. BLAST search against NCBI nr database $\left(\mathrm{E}\right.$-value $=1 \mathrm{e}^{-10}$ ) revealed additional units in BAC clones from T. turgidum subsp.durum [GenBank:EF081027], T. turgidum subsp. dicoccoides [GenBank:EF067844] and H. vulgare [GenBank:DQ871219] with similarities ranging from 70 to $90 \%$. In each sequence, 3 - 5 tandemly organized units were identified.

Repeat composition of rye BESs was compared to 10.8 Mbp sequence of random BESs obtained from wheat chromosome 3B [18] and 2.9 Mbp of wheat D genome sequence derived from a genomic shotgun library of the $\mathrm{D}$ genome progenitor Aegilops tauschii [36]. The frequency of various types of repeats in rye, wheat $B$ and $D$ genomes revealed a close relationship of the rye genome and wheat $\mathrm{B}$ genome (Figure 3). To support this observation, wheat $B$ and $D$ genome sequences were compared to rye genome using RepeatMasker with CrossMatch algoritm. Search of 3B BESs against rye BESs masked $75.4 \%$ 3B BESs with average identity $81.3 \%$. Search of D genome sequences

Table 2: Representation of repetitive element groups on IRS

\begin{tabular}{|c|c|c|c|c|}
\hline & & & Cumulative length (bp) & Fraction of IRS (\%) \\
\hline \multirow[t]{8}{*}{ Class I elements } & & & $\mathrm{I}, 306,78 \mathrm{I}$ & 64.3 \\
\hline & LTR retrotransposons & & $1,275,443$ & 62.8 \\
\hline & & Gypsy & 989,195 & 48.7 \\
\hline & & Copia & 281,937 & 13.9 \\
\hline & & TRIM & 265 & 0.0 \\
\hline & Non-LTR retrotransposons & & 31,338 & 1.5 \\
\hline & & LINE & 23,400 & 1.2 \\
\hline & & SINE & I,877 & 0.1 \\
\hline \multirow[t]{5}{*}{ Class II elements } & & & 100,854 & 5.0 \\
\hline & & CACTA & 90,323 & 4.4 \\
\hline & & Mutator & 1,394 & 0.1 \\
\hline & & MITE & 4,681 & 0.2 \\
\hline & & LITE & 2,753 & 0.1 \\
\hline Unclassified elements & & & 30,340 & 1.5 \\
\hline \multirow[t]{4}{*}{ Other known repeats } & & & 106,376 & 5.2 \\
\hline & Ribosomal genes & & 94,665 & 4.7 \\
\hline & Simple repeats & & 5,049 & 0.2 \\
\hline & Tandem repeats & & 6,662 & 0.3 \\
\hline Known repeats & & & $1,536,587$ & 75.6 \\
\hline Unknown repeats & & & 178,027 & 8.8 \\
\hline Total & & & $\mathrm{I}, 7 \mid 2,364$ & 84.2 \\
\hline
\end{tabular}




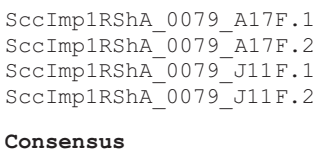

SCCImp1RShA_0079_A17F.1 SCCImp1RShA_0079 A17F.2 ScCImp1RShA 0079 J11F.1 SCCImp1RShA $0079^{-} \mathrm{J} 11 \mathrm{~F} .2$ Consensus

SCCImp1RShA 0079 A17F. SCCImp1RShA $0079^{-}$A17F.2 SCCImp1RShA ${ }^{-} 0079^{-}$J11F.1 SCCImp1RShA_0079_J11F.2 Consensus

SCCImp1RShA 0079 A17F. SCCImp1RShA $0079^{-}$A17F.2 SCCImp1RShA_0079_J11F.1 SCCImp1RShA_0079-J11F.2

Consensus

SCCImp1RShA_0079_A17F.1 SCCImp1RShA_0079_A17F.2 ScCImp1RShA 0079 J11F.1 SCCImp1RShA $0079^{-}$J11F.2

Consensus

SCCImp1RShA_0079_A17F.1 SCCImp1RShA 0079 A17F.2 SCCImp1RShA 0079 J11F.1 SCCImp1RShA $0079^{-}$J11F.2

Consensus

Figure 2

Multiple alignment of four units of COPI repeat. Four units of repeat discovered in BESs ScclmpIRShA_0079_AI7F and ScclmpIRShA_0079_JIIF were aligned with ClustalW [50]. Consensus sequence was calculated from the alignment.

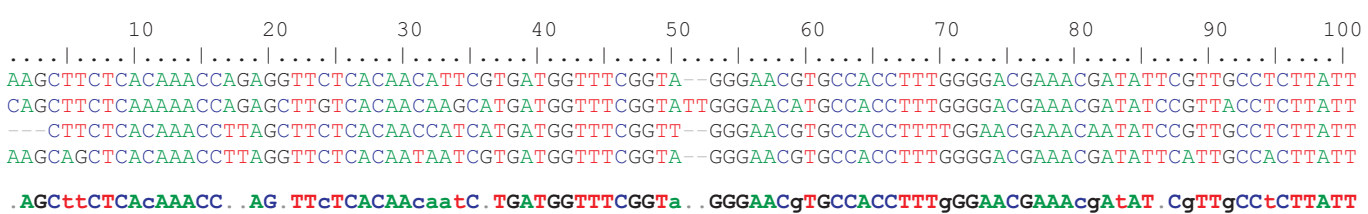

160

170

180

190 GCTTTCAAAAAATTTCTAGTGTCAACATAGAACAATAGGAGTGTTGTGTCTAATTTTGGAATCTTTTGGGGCTCGTTTGGACATTTTTATACATTAAGTG GCTATCAAAAAATTCTAGTGTCAACATAGAAAAACAGGAGTGTTGTGTCTAATTTTGGAATTTTTCAAGGTTCGTTTGGACATTTTTATACATTAACTA GCTTTCAAAATCTTTCGATTGTCAACATAGAACAACATAAGTGTTGTGTTGAATTTTGGAATTTTTCAGGGTTCGTTTGGACATTTTTATACATCAACTA GCTTTAAAAATCTTTCGATTGTCAACATAGAACACCAGGCGTGTTGTGTTCAATTTTGGACTTTTTTGGGGCTCATTTGGACATTTTTATACATTAACTA

GCTtTCAAAA . TTTC . A . TGTCAACATAGAAcAacAggaGTGTTGTGT . AATTTTGGAaTtTTT . . Ggg . TCgTTTGGACATTTTTATACATtAACTa 210
220 $230 \quad 240 \quad 250 \quad 260 \quad 270 \quad 280 \quad 300$ -TTTTTAGGCATTTTATGTGCATAACTCAAATTTGAACTACATGCACATGCTCCAGTGCATATAAACGGGTTGAAAAATCAAATTTGTGTCCTTCGAT GGTTTTCTAGGCATTTTATGTGCATAATTAAAATTTGAACTACGTGCACATGCTCCAGTGCATATAAACAGGTTGAAAAATAAAATATGTGTCCGACGGT GGCTTTCCAGGCATTTTACTCGCATAATTCAAATTTGAACTACATGCACATTCTCGAGTGCATATAAACTGGTTGAAGA-TCAAATATGTGTCCTTGGTT GGTTTTCACGCATTTTATTCACACAATTCAAATTTGAACTACATGCACATTCTCAAGTGCATATAAACTGGTTGAAAAATCAAAT

GGtTTT . AgGCATTTTAt. gCAtAAtTCAAATTTGAACTACaTGCACAT . CTC . AGTGCATATAAAC . GGTTGAAaAATCAAAT . TGTGTCC . . . . T $\begin{array}{lllllllll}310 & 320 & 330 & 340 & 350 & 360 & 370 & 380 & 390\end{array}$ GCATTTTTAGGTCCCATGCAAGAAATGGGAGTGAATTTCAAACACCCTACCACCGTCACTAGGCCGGAAACATCGAGATACCTGGATTTT-AAATTCTAG GCATGCT

GCACGCTTATGTGCCATGCAAGAAATGGGAATGAATTTAAAAAACCCTGCCACCGTCACTCAAACGCAAACATTGAGATACCTGGTTTTTTAAATTCTAG

GCA. . TTA . GT . CCATGCAAGAAATGGGA . TGAATTT . AAA . ACCCT . CCACCGTCACT . . . CG . AAACAT . GAGATACCTGG . TTTTTAAATTCTAG $\begin{array}{lllllllll}410 & 420 & 430 & 440 & 450 & 460 & 470 & 480 & 490\end{array}$ $\ldots|\ldots . \ldots| \ldots|\ldots| \ldots|\ldots| \ldots|\ldots| \ldots|\ldots| \ldots|\ldots| \ldots|\ldots| \ldots|\ldots| \ldots|\ldots| \ldots|\ldots| \ldots|\ldots| \ldots|\ldots|$ TAAATCCAACACTCGTCTAAAATTCATGAAACTTGGCATGCTATCATAGAGCGGTATCAACATGCCGTGGTAAATTTTTTGTCGCATTTGGGGCAGGTTT TAAATCCAAAACTCGTAGAAAATTCGTGAAACTTGGCATGCTATCATGGAGCAGCATCAACATGCCGTGGTAA-TTTTTT--CCCATTTGGGGCAGGTTT TAAATCCAA . ACTCGT . AAAATTC . TGAAACTTGGCATGCTATCAT . GAGC . G . ATCAACATGCCGTGGTAAATTTTTTGTC . CATTTGGGGCAGGTTT GTGTAT GGGTAT

G. GTAT against rye BESs masked only 57.6\% D genome sequence with average identity $79.7 \%$.

\section{Gene content analysis}

Repeat-masked sequences were subjected to a homology search using BLASTN (E-value $=1 e^{-30}$ ) against 960,574 plant PUTs downloaded from PlantGDB $[27,28]$ to identify the transcribed part of the BAC end sequences. The search retrieved 93 hits. Sequences with homology to TErelated proteins were excluded from the analysis. After that, the remaining transcribed part represented 18,256 bp i.e. $0.9 \%$ of the complete sequence set. Assuming the average length of a gene in the family Poaceae to be $2 \mathrm{kbp}$, one can estimate 2,000 genes being present on chromosome arm 1RS and 36,000 genes in the whole rye genome. Forty-one sequences with alignment longer than $200 \mathrm{bp}$ were searched against protein database using BLASTX. Protein with significant homology (E-value $\left.<1 e^{-10}\right)$ was identified for 17 of them. Eleven of them have a putative function (Table 3).

\section{Development and mapping of molecular markers}

Simple sequence repeats (SSRs) were identified in the data set using SciRoKo 3.3 software (see Material and Methods). In total, 216 SSRs were identified with an average length of $19.85 \mathrm{bp}$. The most abundant motifs were trinucleotides, which were found 92-times (Table 4). On average, one microsatellite was found every $9500 \mathrm{bp}$. In addition, a total of 249 sites of insertion of transposable elements were identified in the data set of 2,032,538 Mbp. Thus one may expect one transposable element insertion every $8200 \mathrm{bp}$ in the rye genome. Primer pairs were designed for the 234 identified ISBPs (94.0\%).

Sixty-four ISBP primer pairs were tested for 1 RS specificity. Twelve of them (ora001 - ora012) provided an amplifica- 


\section{Wheat B genome}

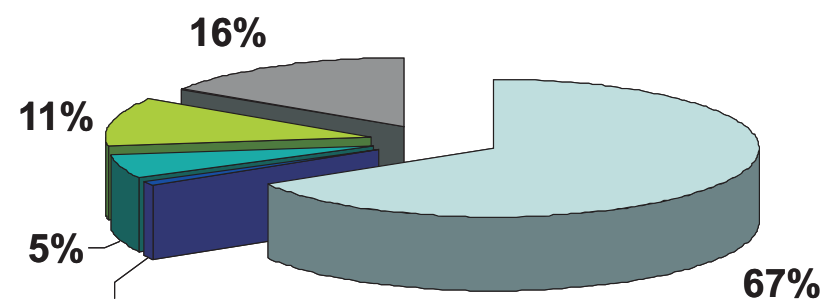

$1 \%$

\section{Wheat D genome}

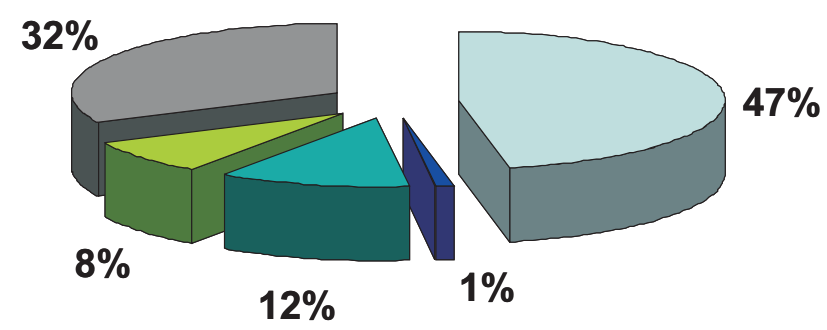

\section{Rye genome}

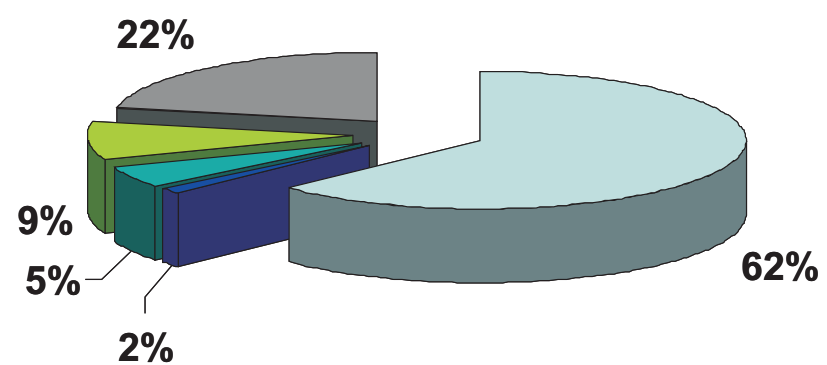

LTR

Non-LTR

DNA trans.

Figure 3

Comparison of rye and wheat $B$ and $D$ genomes. Rye genome is represented by 2 Mbp sequence of IRS-specific BESs. Wheat $B$ genome is represented by $10.8 \mathrm{Mbp}$ sequence of random BES obtained from wheat chromosome 3B [18] and wheat $\mathrm{D}$ genome is represented by $2.9 \mathrm{Mbp}$ sequence derived from a genomic shotgun library of the $\mathrm{D}$ genome progenitor Aegilops tauschii [36]. Note similar repeat composition of the rye and wheat B genomes.

tion product in rye and in the wheat-rye $1 \mathrm{RS}$ addition line but did not show any amplification with wheat DNA and thus were considered 1RS specific (Figure 4). An additional five markers (ora013 - ora017), that amplified a product from the wheat-rye $1 \mathrm{RS}$ addition line, were absent from rye. All 1RS-specific primers are listed in Additional file 1. Finally, ten ISBP markers were found specific for wheat. Gel electrophoresis with PCR products of the remaining 37 primer pairs resulted in bands occurring in both rye and wheat, in a smear, or had no product.

\section{Genomic distribution of the COPI repeat}

FISH with a probe for the COP1 repeat resulted in a signal localized on proximal end of the satellite of chromosome $1 \mathrm{R}$; two other clusters localized proximally on short arms of other two chromosome pairs (Figure 5a). Thus, the

Table 3: Protein homologs of predicted genes discovered in BESs from IRS

\begin{tabular}{|c|c|c|c|c|}
\hline BES & Function & GenBank accession & Organism & E-value \\
\hline ScclmpIRSbA_0223_M04F & Mla-like protein & AAR34459 & Triticum aestivum & $|e-| 2 \mid$ \\
\hline ScclmpIRSbA_0223_AI2R & Cytochrome P450 & AAK38080 & Lolium rigidum & $4 e-96$ \\
\hline ScclmpIRSbA_0I75_BI4R & Putative nucleotide excision repair protein XP-D & NP 001054627 & Oryza sativa & $6 e-96$ \\
\hline ScclmpIRShA_0079_P08R & Putative isoleucin-tRNA ligase & $\overline{N P 001058191}$ & Oryza sativa & $8 e-83$ \\
\hline ScclmpIRSbA_0223_DOIR & Kinase R-like protein & $\overline{\mathrm{AAL} 5107 \mid}$ & Triticum aestivum & $2 e-74$ \\
\hline ScclmplRSbA_0I75_FI7F & MLAI & $\overline{\text { AAG37354 }}$ & Hordeum vulgare & $3 e-60$ \\
\hline ScclmpIRSbA_0223_PI6R & Cytochrome P450 & $\overline{\text { BAD37502 }}$ & Oryza sativa & le-56 \\
\hline ScclmpIRSbA_0I75_G24F & ACC oxidase & $\mathrm{AB} \mid 15735$ & Triticum monococcum & $2 e-63$ \\
\hline ScclmpIRSbA_0I75_M02F & HCBT-like putative defense response protein & AAT38406 & Aegilops tauschii & $5 e-39$ \\
\hline ScclmpIRShA_0I27_EI2R & Cystein proteinase & NP 001062479 & Oryza sativa & $3 e-33$ \\
\hline ScclmpIRSbA_0I75_G23F & Putative tRNA His guanylyltransferase & $\overline{\text { EAY98824 }}$ & Oryza sativa & $8 e-29$ \\
\hline
\end{tabular}


Table 4: Statistics of microsatellites discovered in IRS-specific BESs

\begin{tabular}{lccc}
\hline Motif & Count & Average length (bp) & Count/Mbp \\
\hline Mononucleotide & 19 & 15.89 & 9.35 \\
Dinucleotide & 30 & 29.60 & 14.76 \\
Trinucleotide & 92 & 19.99 & 45.26 \\
Tetranucleotide & 39 & 15.87 & 19.19 \\
Pentanucleotide & 27 & 16.96 & 13.28 \\
Hexanucleotide & 9 & 20.22 & 4.43 \\
\hline Total & 216 & 19.85 & 106.27 \\
\hline
\end{tabular}

COP1 repeat seems to be clustered on three pairs of rye chromosomes. In spite of using blocking DNA prepared from sheared genomic rye DNA, cross-hybridization was detected on all rye chromosomes. FISH in hexaploid wheat revealed dispersed signals over 14 chromosomes (Figure 5b), indicating that COP1 is dispersed in one of the three wheat homoeologous genomes. Reprobing with probes for GAA microsatellites and Afa repeat proved that the repeat is localized on the $\mathrm{D}$ genome chromosomes.
No signal was detected after FISH with the COP1 repeat on barley metaphase chromosomes (Figure 5c).

\section{Discussion}

We generated and analyzed $2 \mathrm{Mbp}$ of BAC end sequences from the short arm of rye chromosome 1 (1RS) accounting for $0.5 \%$ DNA of the arm. This study provides the largest amount of genomic sequence data for rye and allows the first systematic analysis of the DNA sequence composition of the rye genome. Because the BAC clones selected for end sequencing were chosen randomly and originated from BAC libraries constructed with two different restriction enzymes, the BESs produced here are expected to be randomly distributed along the whole chromosome arm. Assuming that there is no or little difference in sequence composition among different rye chromosomes, one can consider this sequence as representative of the whole rye genome. The GC content of $1 \mathrm{RS}(45.9 \%)$ is comparable to $44.5 \%$ and $44 \%$ GC content in wheat (estimated from 37,609 BES downloaded from GenBank [31]) and rice genomes [9], respectively.

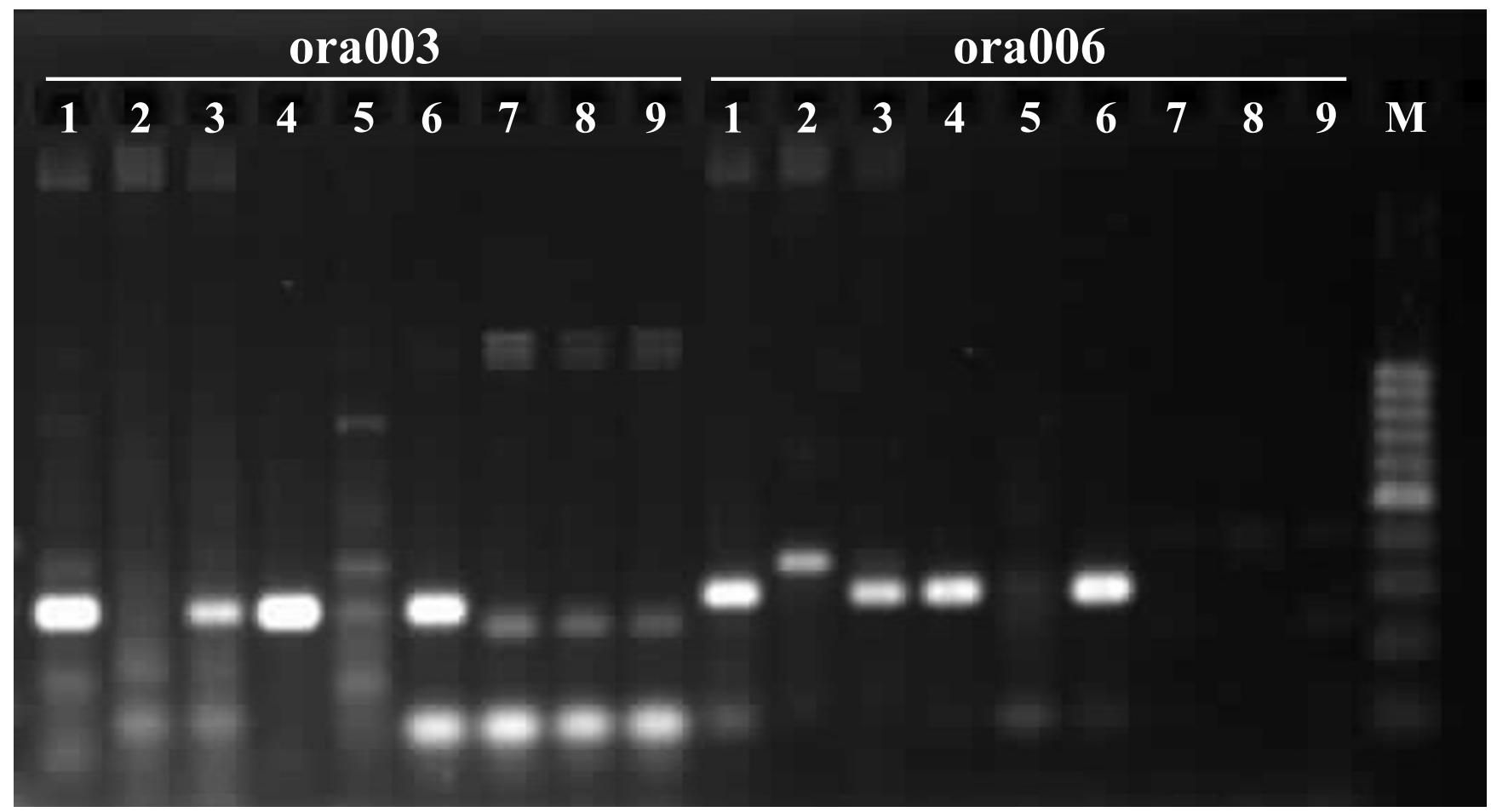

\section{Figure 4}

Example of localization of ISBP markers onchromosome arm IRS. PCR products obtained after amplification with two ISBP primer pairs were visualized on a $2 \%$ agarose gel. Templates were DNA of rye (I), wheat (2), wheat-rye IRS addition (3), flow-sorted chromosome IR (4), and flow-sorted chromosomes 2R - 7R (5) were used for testing marker specificity. BAC pools of plate ScclmpIRShA_0079 (6), ScclmpIRShA_0I 27 (7), ScclmpIRSbA_0I75 (8), and ScclmpIRSbA_0223 served to estimate frequency of ISBP sites on IRS and as positive control. Both markers were positive in rye, the wheat-rye IRS addition line and in flow-sorted chromosome IR and negative in wheat and flow-sorted chromosomes $2 R-7 R$ and thus were considered IRS specific. M - 100 bp DNA ladder. 

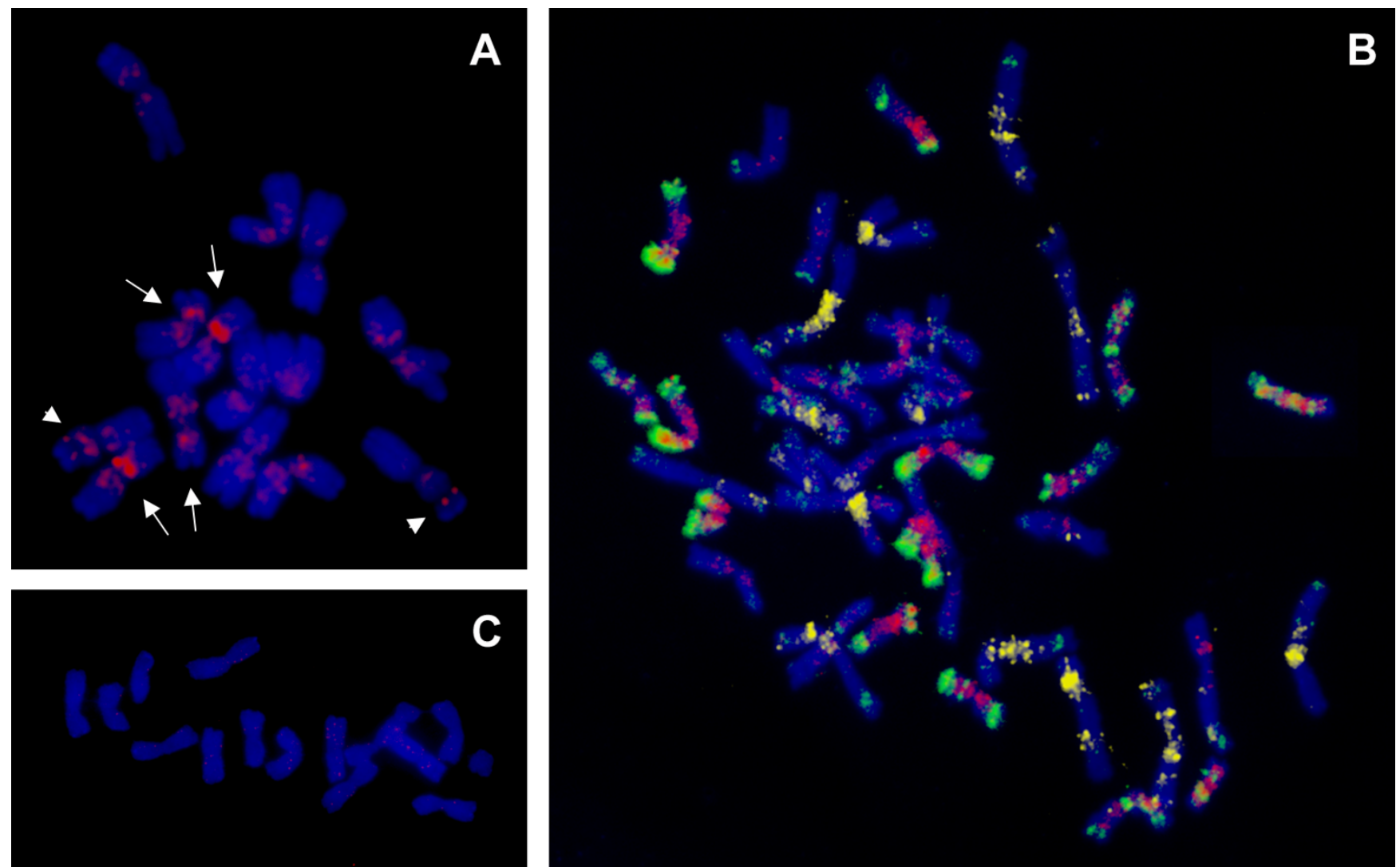

Figure 5

FISH localization of COPI repeat on mitotic metaphase chromosomes. (A) COPI repeat (red) is localized on three chromosome pairs of rye including the short arm of chromosome IR (arrowheads). (B) In hexaploid wheat, COPI shows dispersed signals on 14 chromosomes (red), belonging to the D genome, which was identified using probes for GAA microsatellites (yellow) and Afa family repeat (green). (C) No signal was detected after FISH with COPI on barley chromosomes. Chromosomes were counterstained with DAPI (blue).

The observed content of repetitive sequences $(84.2 \%)$ is lower than expected and is similar to that found in the wheat B genome (85.9\%) by Paux et al. [18]. As indicated above, rye has a significantly higher $1 \mathrm{Cx}$ value than bread wheat. Thus, assuming an equal number of genes, the rye genome should contain more repeats than the wheat genome. In fact, using Cot analysis, Flavell et al. [8] estimated the content of repetitive elements in rye genome to be of about $92 \%$. Our low estimate could be due to insufficient representation of rye repeats in databases that were used in our analyses. For example, the TREPtotal database, which showed most of the significant matches, comprises only 39 entries of rye repeats. This limited information, compared to wheat and barley with 663 and 554 elements, respectively (in the same database), could result is short sequence alignments and hence underestimation of the amount of repeated DNA in rye BES. As expected, Class I elements (retrotransposons) were the most abundant repetitive fraction in the rye genome similar to what was found for the wheat and maize genomes [18,37].
A comparison of the frequency of various types of repeats in genomes of rye and wheat $\mathrm{B}$ and $\mathrm{D}$ genomes suggested a greater similarity between the rye and wheat $\mathrm{B}$ genome than between the rye and wheat $\mathrm{D}$ genome. This close relationship of rye and wheat $\mathrm{B}$ genome was also supported at the sequence level. It is interesting to note that rye and the putative $\mathrm{B}$ genome progenitor Aegilops speltoides have the same mating system, both being outcrossers. Moreover, the $\mathrm{B}$ genome is largest of the three homeoelogous wheat genomes and similar in size to the rye genome. On the other hand, the similarity in repeat composition between both genomes may simply reflect similar trends in the mode of their expansion via the LTRretrotransposon activation.

Until now, the lack of sequence data did not permit estimation of the number of genes in rye. By analyzing $2 \mathrm{Mbp}$ sequence from chromosome arm 1RS, we estimate 2,000 genes being present on 1RS and thus 36,000 genes in the rye genome. This first estimate for rye is consistent with 
the predicted gene numbers in other plants. Most recent estimate for gene number in A. thaliana genome is 33,000 (TAIR8 release) [38], the current TIGR rice genome annotation (Release 5) [39] estimates 41,000 genes in rice genome. Both numbers are close to our prediction for rye.

In addition to the analysis of the rye genome composition, we used BAC end sequences for marker development. There is still a low density of markers available for rye genome and additional markers are urgently needed. Development of a genetic linkage map of rye with 183 markers was reported by Korzun et al. [40]. Bednarek et al. [41] presented a genetic map of rye containing 480 markers including 200 RFLPs, 179 AFLPs, 88 RAPDs and 13 proteins. Khlestkina et al. [42] mapped 99 SSRs derived from EST sequences (SSR-ESTs), nine of which mapped to chromosome 1R. Several attempts were made to transfer SSR and/or EST-SSR markers from wheat and barley into rye [43-45]. Recently Varshney et al. [46] succeeded in transferring and mapping 12 barley SNP markers in rye. Thus, to date the density of markers is quite low and does not allow efficient map based cloning or MAS in rye.

This work presents a method for targeted development of molecular markers from specific parts of the rye genome using the 1RS chromosome arm as a case study. Until now, there are only a few markers available for this critical part of the rye genome. This hampers marker assisted breeding not only in rye, but also in wheat where the markers from 1RS would permit selection of lines with introgressions of desired parts of 1RS without the harmful genes. We have developed twelve new ISBPs markers for $1 \mathrm{RS}$ and designed almost 200 additional primer pairs for potential ISBP markers that remain to be tested. If needed, generation of additional BAC end sequences from 1RS is possible. In addition to the development of new ISBP markers, BES containing microsatellites were used to develop new 1RS-specific SSR markers. Out of the 63 tested microsatellites that were tested, 21 were specific for $1 \mathrm{RS}$ [47]. Thus, in total 33 1RS-specific markers were obtained using our strategy. On the other hand, Badnarek et al. [41] isolated 198 AFLP and RAPD markers from genomic DNA and only 29 markers were specific for 1RS. This clearly demonstrates the efficiency of the targeted approach.

One of the potential problems in targeted marker development from the 1RS-specific BAC library is the contamination with clones from wheat DNA. This could explain the specificity of amplification for several ISBP markers with wheat DNA. As the 1RS arm was flow-sorted from a wheat-rye ditelosomic addition line, contamination by fragments of wheat chromosomes cannot be avoided. However, the contamination of the 1RS BAC library was estimated to be only 14\% [15] and thus should not seri- ously compromise the efficiency of marker development. Further improvement could be achieved by selecting BAC clones from contigs after fingerprinting the library, as the contigs originate from the chromosome of interest while the infrequent contaminating BACs remain as singletons.

The discovery of five ISBP markers, which were specific only for 1 RS maintained in the wheat-rye ditelosomic addition line and were not found in diploid rye, was unexpected. Although the same cultivars (i.e. Chinese Spring and Imperial) were used for the development of the 1RS wheat-rye addition line as well as for marker testing, these insertion sites were absent from rye and wheat. This suggests that some mobile elements were activated only after the addition of $1 \mathrm{RS}$ telocentric to wheat, most probably as a consequence of interspecific hybridization. Among the five activated elements three are retrotransposons (Copialike, LINE and SINE) and two are DNA transposons (MITE and Mutator). Liu and Wendel [48] and Shan et al. [49] observed similar activation of both classes of transposable elements after a hybridization of cultivated (Oryza sativa) and wild (Zizania latifolia) rice.

\section{Conclusion}

This work provides the first insights into the composition of the rye genome and its chromosome arm 1RS, in particular. We demonstrate that the use of chromosome armspecific BAC libraries facilitates the analysis of complex plant genomes by targeting particular genomic regions as well as by developing molecular markers for these regions. New molecular markers from 1RS should help in saturating the genetic map of 1RS, and aid marker assisted breeding and gene cloning.

\section{Abbreviations}

1RS: short arm of rye chromosome 1; BAC: bacterial artifical chromosome; BES: BAC end sequence; ISBP: Insertion Site Based Polymorphism; PUT: PlantGDB-assembled Unique Transcripts

\section{Authors' contributions}

$\mathrm{JB}, \mathrm{EP}$ and RK participated in DNA sequence analysis. JB and $\mathrm{MH}$ mapped newly isolated ISBP markers. DK performed the FISH experiments. PS sorted chromosomes using flow cytometry. JŠ and HŠ made an intellectual contribution to the concept of the experiment. CT sequenced BAC ends. JB drafted the manuscript. TL and CF revised manuscript critically for important intellectual content, JD conceived and supervised the project and prepared the final version of the manuscript. All authors read and approved the final manuscript. 


\section{Additional material}

\section{Additional file 1}

List of $1 R S$-specific ISBP markers

Click here for file

[http://www.biomedcentral.com/content/supplementary/1471-

2229-8-95-S1.pdf]

\section{Acknowledgements}

We dedicate this paper to Dr. Jiří Velemínský, former director of the Institute of Experimental Botany (IEB, Prague), who contributed significantly to the development of the Olomouc Research Centre of IEB and who passed away during the preparation of this manuscript. We thank Professor A. J. Lukaszewski and Dr. P. Martínek for providing seed stocks and M. Sekerová, Bc., R. Tušková and $\mathrm{H}$. Tvardíková for excellent technical assistance.

This work was supported by research grants No. 52I/06/P4I 2 and 52I/05/ $\mathrm{HOI} 3$ from the Czech Science Foundation and grant No. LC06004 from the Ministry of Education, Youth and Sports of the Czech Republic.

\section{References}

I. Rabinovich SV: Importance of wheat-rye translocations for breeding modern cultivars of Triticum aestivum L. (Reprinted from Wheat: Prospects for global improvement, 1998). Euphytica 1998, 100:323-340.

2. Schlegel R, Korzun V: About the origin of IRS. IBL wheat-rye chromosome translocations from Germany. Plant Breeding 1997, I | 6:537-540.

3. Burnett C], Lorenz KJ, Carver BF: Effects of the IB/IR translocation in wheat on composition and properties of grain and flour. Euphytica 1995, 86:।59-166.

4. Mago R, Miah H, Lawrence G], Wellings CR, Spielmeyer W, Bariana HS, Mclntosh RA, Pryor AJ, Ellis JG: High-resolution mapping and mutation analysis separate the rust resistance genes Sr3 I, Lr26 and Yr9 on the short arm of rye chromosome I. Theoretical and Applied Genetics 2005, I I 2:4 I-50.

5. Voylokov AV, Korzun V, Borner A: Mapping of three self-fertility mutations in rye (Secale cereale L.) using RFLP, isozyme and morphological markers. Theoretical and Applied Genetics 1998, 97:147-153.

6. Graybosch RA: Uneasy unions: Quality effects of rye chromatin transfers to wheat. Journal of Cereal Science 2001, 33:3-16.

7. Genes, markers and linkage data of rye (Secale cereale L.) 6th updated inventory [http://www.desicca.del Rye\%20gene\%20map/]

8. Flavell RB, Bennett MD, Smith JB, Smith DB: Genome size and the proportion of repeated nucleotide sequence DNA in plants. Biochem Genet 1974, I 2:257-269.

9. Goff SA, Ricke D, Lan TH, Presting G, Wang R, Dunn M, Glazebrook J, Sessions A, Oeller $P$, Varma $H$, et al.: A draft sequence of the rice genome (Oryza sativa L. ssp. japonica). Science 2002, 296:92-100

10. Matsumoto T, Wu JZ, Kanamori H, Katayose Y, Fujisawa M, Namiki $\mathrm{N}$, Mizuno $\mathrm{H}$, Yamamoto K, Antonio BA, Baba T, et al.: The mapbased sequence of the rice genome. Nature 2005, 436:793-800.

II. Yu J, Hu SN, Wang J, Wong GKS, Li SG, Liu B, Deng YJ, Dai L, Zhou $Y$, Zhang $X Q$, et al:: A draft sequence of the rice genome (Oryza sativa L. ssp indica). Science 2002, 296:79-92.

12. Dolezel J, Kubalakova M, Bartos J, Macas J: Flow cytogenetics and plant genome mapping. Chromosome Res 2004, I 2:77-9I.

13. Dolezel J, Kubalakova M, Paux E, Bartos J, Feuillet C: Chromosomebased genomics in the cereals. Chromosome Res 2007, 1 5:51-66.

14. Kubalakova M, Valarik M, Bartos J, Vrana J, Cihalikova J, Molnar-Lang M, Dolezel J: Analysis and sorting of rye (Secale cereale L.) chromosomes using flow cytometry. Genome 2003, 46:893-905.

15. Simkova H, Safar J, Suchankova P, Kovarova P, Bartos J, Kubalakova $M$, Janda J, Cihalikova J, Mago R, Lelley $\mathrm{T}$, et al.: A novel resource for genomics of Triticeae: BAC library specific for the short arm of rye (Secale cereale L.) chromosome IR (IRS). BMC Genomics 2008, 9:237.

16. Kelley JM, Field CE, Craven MB, Bocskai D, Kim UJ, Rounsley SD, Adams MD: High throughput direct end sequencing of BAC clones. Nucleic Acids Research 1999, 27: I539-I546.

17. Shultz JL, Kazi S, Bashir R, Afzal JA, Lightfoot DA: The development of BAC-end sequence-based microsatellite markers and placement in the physical and genetic maps of soybean. Theoretical and Applied Genetics 2007, I | 4: 108 |-1090.

18. Paux E, Roger D, Badaeva E, Gay G, Bernard M, Sourdille P, Feuillet $C$ : Characterizing the composition and evolution of homoeologous genomes in hexaploid wheat through BAC-end sequencing on chromosome 3B. Plant Journal 2006, 48:463-474.

19. Safar J, Bartos J, Janda J, Bellec A, Kubalakova M, Valarik M, Pateyron $\mathrm{S}$, Weiserova J, Tuskova R, Cihalikova J, et al.: Dissecting large and complex genomes: flow sorting and BAC cloning of individual chromosomes from bread wheat. Plant Journal 2004, 39:960-968.

20. Chou $\mathrm{HH}$, Holmes $\mathrm{MH}$ : DNA sequence quality trimming and vector removal. Bioinformatics 2001, I7:1093-I 104.

21. TREP, the Triticeae Repeat Sequence Database [http:// wheat.pw.usda.gov/ITMI/Repeats/]

22. Wicker T, Matthews DE, Keller B: TREP: a database for Triticeae repetitive elements. Trends in Plant Science 2002, 7:56I-562.

23. Repbase [http://www.girinst.org/repbase/index.html]

24. Jurka J, Kapitonov VV, Pavlicek A, Klonowski P, Kohany O, Walichiewicz J: Repbase update, a database of eukaryotic repetitive elements. Cytogenetic and Genome Research 2005, I I 0:462-467.

25. The TIGR Plant Repeat Databases [http://www.tigr.org/tdb/ e2kl/plant.repeats/]

26. Ouyang S, Buell CR: The TIGR Plant Repeat Databases: a collective resource for the identification of repetitive sequences in plants. Nucleic Acids Research 2004, 32:D360-D363.

27. PlantGDB - Resources for Plant Comparative Genomics [http://www.plantgdb.org/]

28. Dong Q, Lawrence CJ, Schlueter SD, Wilkerson MD, Kurtz S, Lushbough C, Brendel V: Comparative plant genomics resources at PlantGDB. Plant Physiol 2005, I39:610-618.

29. RepeatMasker [http://www.repeatmasker.org/]

30. Altschul SF, Madden TL, Schaffer AA, Zhang JH, Zhang Z, Miller W, Lipman DJ: Gapped BLAST and PSI-BLAST: a new generation of protein database search programs. Nucleic Acids Research 1997, 25:3389-3402.

31. GenBank [http://www.ncbi.nlm.nih.gov/Genbank/index.html]

32. Kofler R, Schlotterer C, Lelley T: SciRoKo: a new tool for whole genome microsatellite search and investigation. Bioinformatics 2007, 23:1683-1685.

33. Rozen S, Skaletsky HJ: Primer3 on the WWW for general users and for biologist programmers. In Bioinformatics methods and protocols: Methods in molecular biology Edited by: Krawetz S, Misener S. Totowa: Humana Press; 2000:365-386.

34. Masoudi-Nejad A, Nasuda S, Mclntosh RA, Endo TR: Transfer of rye chromosome segments to wheat by a gametocidal system. Chromosome Research 2002, 10:349-357.

35. Kubalakova M, Kovarova P, Suchankova P, Cihalikova J, Bartos J, Lucretti S, Watanabe N, Kianian SF, Dolezel J: Chromosome sorting in tetraploid wheat and its potential for genome analysis. Genetics 2005, I 70:823-829.

36. Li WL, Zhang P, Fellers JP, Friebe B, Gill BS: Sequence composition, organization, and evolution of the core Triticeae genome. Plant Journal 2004, 40:500-5II.

37. Haberer G, Young S, Bharti AK, Gundlach H, Raymond C, Fuks G, Butler E, Wing RA, Rounsley S, Birren B, et al.: Structure and architecture of the maize genome. Plant Physiology 2005, 139:1612-1624.

38. The Arabidopsis Information Resource [http://www.arabidop sis.org/]

39. TIGR Rice Genome Annotation [http://www.tigr.org/tdb/e2kl/ osal/]

40. Korzun V, Malyshev S, Voylokov AV, Borner A: A genetic map of rye (Secale cereale L.) combining RFLP, isozyme, protein, microsatellite and gene loci. Theoretical and Applied Genetics 200 I, 102:709-7|7.

4I. Bednarek PT, Masojc P, Lewandowska R, Myskow B: Saturating rye genetic map with amplified fragment length polymorphism 
(AFLP) and random amplified polymorphic DNA (RAPD) markers. J Appl Genet 2003, 44:2I-33.

42. Khlestkina EK, Ma HMT, Pestsova EG, Roder MS, Malyshev SV, Korzun V, Borner A: Mapping of $\mathbf{9 9}$ new microsatellite-derived loci in rye (Secale cereale L.) including 39 expressed sequence tags. Theoretical and Applied Genetics 2004, 109:725-732.

43. Kuleung C, Baenziger PS, Dweikat I: Transferability of SSR markers among wheat, rye, and triticale. Theoretical and Applied Genetics 2004, 108: I |47-I I 50.

44. Varshney RK, Sigmund R, Borner A, Korzun V, Stein N, Sorrells ME, Langridge $P$, Graner A: Interspecific transferability and comparative mapping of barley EST-SSR markers in wheat, rye and rice. Plant Science 2005, 168:195-202.

45. Zhang LY, Bernard M, Leroy P, Feuillet C, Sourdille P: High transferability of bread wheat EST-derived SSRs to other cereals. Theoretical and Applied Genetics 2005, I I I:677-687.

46. Varshney RK, Beier U, Khlestkina EK, Kota R, Korzun V, Graner A, Borner A: Single nucleotide polymorphisms in rye (Secale cereale L.): discovery, frequency, and applications for genome mapping and diversity studies. Theoretical and Applied Genetics 2007, I | 4: I I05- I I I6.

47. Kofler R, Bartos J, Gong L, Stift G, Suchankova P, Simkova H, Berenyi M, Burg K, Dolezel J, Lelley T: Development of microsatellite markers specific for the short arm of rye (Secale cereale L.) chromosome I. Theor Appl Genet 2008, I I 7(6):915-926.

48. Liu B, Wendel JF: Retrotransposon activation followed by rapid repression in introgressed rice plants. Genome 2000, 43:874-880.

49. Shan XH, Liu ZL, Dong ZY, Wang YM, Chen Y, Lin XY, Long LK, Han FP, Dong YS, Liu B: Mobilization of the active MITE transposons $\mathrm{mPing}$ and Pong in rice by introgression from wild rice (Zizania latifolia Griseb.). Molecular Biology and Evolution 2005, 22:976-990.

50. Chenna R, Sugawara H, Koike T, Lopez R, Gibson TJ, Higgins DG, Thompson JD: Multiple sequence alignment with the Clustal series of programs. Nucleic Acids Research 2003, 31 :3497-3500.

5I. Dolezel J, Greilhuber J, Lucretti S, Meister A, Lysak MA, Nardi L, Obermayer R: Plant genome size estimation by flow cytometry: Inter-laboratory comparison. Annals of Botany 1998, 82(Suppl. A): 17-26.

52. Bennett MD, Smith JB: Nuclear DNA amounts in angiosperms. Philos Trans R Soc Lond B Biol Sci 1976, 274:227-274.

53. Greilhuber J, Dolezel J, Lysak MA, Bennett MD: The origin, evolution and proposed stabilization of the terms "genome size' and ' $C$-value' to describe nuclear DNA contents. Annals of Botany $2005,95: 255-260$.
Publish with Bio Med Central and every scientist can read your work free of charge

"BioMed Central will be the most significant development for disseminating the results of biomedical research in our lifetime. "

Sir Paul Nurse, Cancer Research UK

Your research papers will be:

- available free of charge to the entire biomedical community

- peer reviewed and published immediately upon acceptance

- cited in PubMed and archived on PubMed Central

- yours - you keep the copyright
BioMedcentral 